\title{
DISTRIBUCIÓN ACTUALIZADA Y FENOLOGÍA DE TRICHOPODA PENNIPES (FABRICIUS, 1781) (DIPTERA: TACHINIDAE) EN LA PENÍNSULA IBÉRICA
}

\author{
Antonio Ricarte ${ }^{1, *}$, Piluca Álvarez Fidalgo² \& Marián Álvarez Fidalgo ${ }^{3}$ \\ 1,* Centro Iberoamericano de la Biodiversidad (CIBIO), Universidad de Alicante, \\ Carretera de San Vicente s/n, 03690 San Vicente del Raspeig, Alicante, España. \\ ORCID iD: https://orcid.org/0000-0003-2298-981X - *Autor para correspondencia: ricarte24@gmail.com \\ ${ }^{2} \mathrm{C} /$ Corazón de María, 7-5E, 28002 Madrid, España. \\ ORCID iD: https://orcid.org/0000-0002-5330-3078 - Email: pilucaaf@gmail.com \\ ${ }^{3}$ Avda. del Cristo, 53-5D, 33006 Oviedo, Asturias, España. \\ ORCID iD: http://orcid.org/0000-0002-3313-1467-Email: madamcoolpix@gmail.com
}

\section{RESUMEN}

El taquínido norteamericano Trichopoda pennipes (Fabricius, 1781) es una especie parasitoide introducida en Europa, a consecuencia de su uso en el control de hemípteros plaga como Nezara viridula (Linnaeus, 1758). La captura de dos ejemplares en el municipio de Alicante (España) condujo a una revisión exhaustiva de la bibliografía e información disponible en bases de datos en línea sobre esta especie, con el fin de estudiar su distribución y fenología en el ámbito ibero-balear. El número de cuadrículas UTM de $10 \times 10 \mathrm{~km}$ en las que se ha detectado T. pennipes se incrementa en un $90 \%$ con respecto a lo publicado hasta la fecha, con una gran cantidad de referencias fotográficas frente a otro tipo de citas (82:7). A través de este estudio, la Península lbérica se convierte en la región europea donde está mejor caracterizada la distribución y fenología de esta especie exótica. Se pone de relieve la importancia que los bancos de datos fotográficos y la ciencia ciudadana tienen para el conocimiento de la biodiversidad.

Palabras clave: Diptera, Tachinidae, especie exótica, parasitoide, polinizadores, distribución, fenología, España, Portugal, Alicante

\section{ABSTRACT}

Updated distribution and phenology of Trichopoda pennipes (Fabricius, 1781) (Diptera: Tachinidae) in the Iberian Peninsula

The North American tachinid fly Trichopoda pennipes (Fabricius, 1781) is a parasitoid species introduced in Europe as a consequence of its use in the control of pest bugs such as Nezara viridula (Linnaeus, 1758). After the capture of two specimens in Alicante municipality (Spain) an exhaustive revision of all bibliography and information at online databases on this species were carried out with the aim of studying its distribution and phenology in the lberian-Balearic context. The number of $10 \times 10$ UTM squares where T. pennipes is found increases up to $90 \%$ with respect to what has been published to date, with a great majority of photographic records in relation to other kind of data (82:7). The Iberian Peninsula becomes the best-known European region in terms of this exotic-species distribution and phenology. Once again, photographic databases and citizen science have proven relevant to biodiversity knowledge.

Key words: Diptera, Tachinidae, exotic species, parasitoid, pollinators, range, phenology, Spain, Portugal, Alicante

Cómo citar este artículo/Citation: Ricarte, A., Álvarez Fidalgo, P. \& Álvarez Fidalgo, M. 2020. Distribución actualizada y fenología de Trichopoda pennipes (Fabricius, 1781) (Diptera: Tachinidae) en la Península Ibérica. Graellsia, 76(2): e116. https://doi.org/10.3989/graellsia.2020.v76.271

Copyright: (C) 2020 SAM \& CSIC. This is an open-access article distributed under the terms of the Creative Commons Attribution 4.0 International (CC BY 4.0) License. 


\section{Introducción}

Los Tachinidae (Diptera: Oestroidea) constituyen una de las familias de dípteros de mayor importancia ecológica, debido a su forma de vida endoparasitoide, mediante la cual contribuyen al control natural de grupos de insectos como Hemiptera y larvas de Lepidoptera, entre otros, algunos de los cuales son causantes de plagas (Stireman et al., 2006; Grenier, 1988). Los taquínidos son un grupo de dípteros muy diverso, con más de 8500 especies descritas (Marshall, 2012). Los adultos se caracterizan por la presencia de un subescudete bien desarrollado (con las únicas excepciones de Litophasia Girschner, 1887 y Cinochira Zetterstedt, 1845), presencia de cerdas en la hipopleura (con la excepción de Oxyphyllomyia Villeneuve, 1937) y, en un elevado número de especies, por presentar una cantidad variable de cerdas fuertes y largas, sobre todo en el abdomen (Tschorsnig \& Richter, 1998).

Phasiinae es la subfamilia de taquínidos con menor número de especies y en ella se incluyen muchas sin las largas cerdas características de esta familia. Todos los Phasiinae conocidos son parasitoides de Heteroptera (O'Hara, 2008), siendo Trichopoda pennipes (Fabricius, 1781) de las especies más representativas. Esta especie, nativa de Norteamérica, es fácilmente reconocible por la llamativa hilera de escamas alargadas y planas dispuestas sobre la tibia posterior, así como por la coloración de su cuerpo: ojos pardo-rojizos, alas oscuras con el margen posterior sub-hialino y tórax dorado, con cuatro bandas longitudinales oscuras y lateralmente grises. Los machos presentan el abdomen y la base de las alas anaranjados, mientras que las hembras tienen el abdomen desde completamente negro a anaranjado, con los últimos dos terguitos más o menos oscurecidos, así como la base de las alas frecuentemente oscurecida (Fig. 1) (Worthley, 1924).

Los adultos de $T$. pennipes son nectívoros y pueden polinizar las plantas que visitan. Suelen observarse en

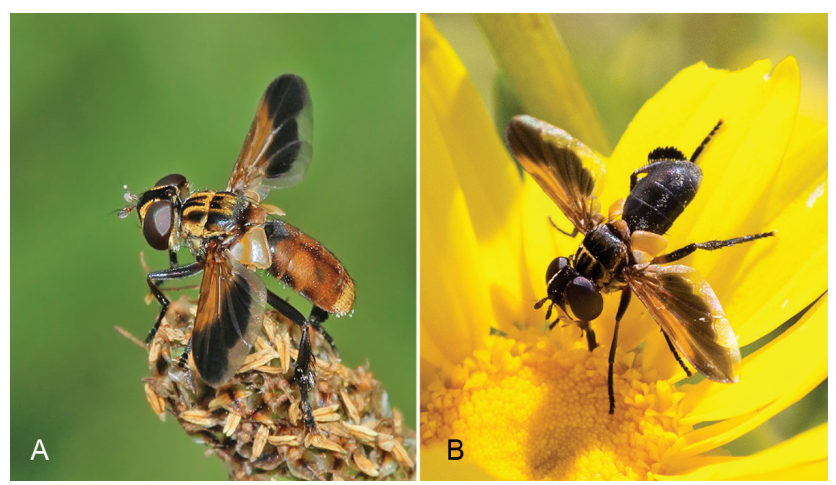

Fig. 1.- Trichopoda pennipes (Fabricius, 1781): macho, Soto del Barco (Asturias) (foto: P. Álvarez Fidalgo); (b) hembra, Altea (Alicante) (foto: A. M. Moreno).

Fig. 1.- Trichopoda pennipes (Fabricius, 1781): male, Soto del Barco (Asturias) (photo: P. Álvarez Fidalgo); (b) female, Altea (Alicante) (photo: A. M. Moreno). días soleados sobrevolando las flores o posados con las alas extendidas (Worthley, 1924). En sus hábitats de origen, se sabe que visitan flores de Anacardiaceae, Apiaceae, Asclepiadaceae, Asteraceae, Caprifoliaceae, Euphorbiaceae, Lamiaceae, Liliaceae, Polygonaceae y Rutaceae, entre otras (Tillman, 2017; Tooker et al., 2006). Las larvas de T. pennipes pasan el invierno dentro del huésped, y los imagos emergen en la siguiente primavera o principios del verano, si bien en condiciones climáticas óptimas pueden presentar dos o tres generaciones al año (Pétremand et al., 2015).

Trichopoda pennipes es conocida por su preferencia hacia la chinche plaga Nezara viridula (Linnaeus, 1758) (Pentatomidae), pudiendo parasitoidizar tanto adultos como ninfas de último estado. Por ello, T. pennipes es utilizada como agente de control biológico de estas chinches (Pilkay et al., 2014). Precisamente, la exportación como agente de biocontrol fuera de su territorio de origen, ha propiciado su introducción accidental en diversos lugares del planeta, incluyendo Europa. Fand et al. (2013) recopilaron un listado de enemigos naturales de diferentes plagas de todo el mundo introducidos accidentalmente fuera de sus países de origen, incluyendo a $T$. pennipes como especie introducida en Italia (Colazza et al., 1996; Salerno et al., 2002). Sin embargo, años antes de la mencionada recopilación, Peris (1998) y Tschorsnig et al. (2000) citan esta especie por primera vez en España de la provincia de Gerona y, posteriormente, Catalán \& Verdú (2005) de la provincia de Valencia. También se ha citado en otros países mediterráneos como Francia (Tschorsnig et al., 2000), Eslovenia (de Groot et al., 2007), Croacia (Bystrowski, 2012), Albania (Skuhravá et al., 2010) e Israel (Freidberg et al., 2011), así como en Holanda (Zeegers, 2010) y Portugal (Almeida et al., 2017).

El hallazgo reciente de esta especie en el municipio de Alicante condujo a investigar la dimensión real de la distribución de esta especie introducida en la Península, ya que los datos existentes en la bibliografía especializada y en colecciones entomológicas parecen no reflejar su verdadera distribución geográfica. Por tanto, este trabajo tiene como objetivo actualizar la información sobre la presencia de $T$. pennipes en la región ibero-balear, utilizando, principalmente, fotografías de campo disponibles en bases de datos y plataformas de ciencia ciudadana.

\section{Material y métodos}

Durante un muestreo de sírfidos (Diptera: Syrphidae) realizado en otoño de 2019 en las proximidades del Edifico de Nuevos Institutos de la Universidad de Alicante (Fig. 2), dos ejemplares de la especie introducida $T$. pennipes fueron recolectados, mediante manga entomológica. Los ejemplares fueron depositados en la Colección Entomológica de la Universidad de Alicante (CEUA), alojada en el instituto CIBIO. 
Para recabar información actualizada sobre la distribución de esta especie en el ámbito peninsular, se revisaron fuentes, tanto bibliográficas (Peris, 1998; Tschorsnig et al., 2000; Carbonell, 2013; Almeida et al., 2017) como de bases de datos en línea: Biodiversidad Virtual (2020), Diptera.info (2020), iNaturalist (2020) y Observation.org (2020); GBIF (2020) también fue consultada, pero todos los registros encontrados ya estaban recogidos en las otras bases de datos anteriormente mencionadas. Todas las localidades que ofrecían información completa y detallada de un registro de $T$. pennipes, y que se consideraron fiables, bien porque el registro fue confirmado por los autores de este trabajo (a partir de las fotografías) o bien por la naturaleza de la fuente, fueron recopiladas en la Tabla 1. Esta tabla recoge todas las cuadrículas UTM de $10 \times 10 \mathrm{~km}$ donde la especie ha sido detectada. En los casos en que se dispone de más de una cita

Tabla 1.- Cuadrículas UTM $10 \times 10 \mathrm{~km}$ donde ha sido registrada Trichopoda pennipes (Fabricius, 1781) en la Península Ibérica y Baleares. Los datos se han obtenido de la plataforma Biodiversidad Virtual, salvo que se indique lo contrario: (1) este trabajo; (2) Flickr; (3) Peris (1998); (4) Tschorsnig et al. (2000); (5) Carbonell (2013); (6) Observation.org; (7) iNaturalist.org; (8) Catalán y Verdú (2005); (9) Almeida et al. (2017); (10) Diptera.info.

Table 1.- UTM squares of $10 \times 10 \mathrm{~km}$ where Trichopoda pennipes (Fabricius, 1781) has been detected in the lberian Peninsula and Balearic Islands. The data have been obtained from the web site Biodiversidad Virtual, unless otherwise stated: (1) this work; (2) Flickr; (3) Peris (1998); (4) Tschorsnig et al. (2000); (5) Carbonell (2013); (6) Observation.org; (7) iNaturalist. org; (8) Catalán y Verdú (2005); (9) Almeida et al. (2017); (10) Diptera.info.

\begin{tabular}{|c|c|c|c|c|c|c|}
\hline \multicolumn{7}{|l|}{ ESPAÑA } \\
\hline UTM & Provincia & Localidad & Altitud & Fecha & Autoría & Fuente \\
\hline 30SYH46 & Alicante & Villajoyosa & $44 \mathrm{~m}$ & $24-X-2012$ & Daniel García & Foto \\
\hline 30SXH87 & Alicante & Villena & $486 \mathrm{~m}$ & $28-\mid X-2014$ & David Molina & Foto \\
\hline 30SYH57 & Alicante & Altea & $29 \mathrm{~m}$ & $15-I V-2019$ & Ángel Mario Moreno & Foto \\
\hline 30SYH15 & Alicante & Alicante & $87 \mathrm{~m}$ & $1-X-2019$ & Antonio Ricarte & Captura $^{(1)}$ \\
\hline 30SVF96 & Almería & Adra & $159 \mathrm{~m}$ & $6-\mathrm{VIII}-2011$ & Francisco Rodríguez & Foto \\
\hline 30SWF37 & Almería & Vicar & $342 \mathrm{~m}$ & $16-\mathrm{VIIII}-2012$ & Francisco Rodríguez & Foto \\
\hline 30SWF16 & Almería & Ejido & $48 \mathrm{~m}$ & 3-VIII-2014 & Francisco Rodríguez & Foto \\
\hline 30SWF19 & Almería & Fondon & $801 \mathrm{~m}$ & 9-VIII-2017 & Francisco Rodríguez & Foto \\
\hline зотUP30 & Asturias & Zardón (Cangas de Onís) & $168 \mathrm{~m}$ & $16-\mathrm{VIIII}-2015$ & Nacho Noval & Foto \\
\hline 29TOJ32 & Asturias & Soto del Barco & $6 \mathrm{~m}$ & $15-X-2017$ & Piluca Álvarez & Foto \\
\hline 31SDE70 & Baleares & Sóller & $58 \mathrm{~m}$ & $22-\mathrm{VI}-2013$ & Juan Bibiloni & Foto \\
\hline 31TDG11 & Barcelona & Mura & $482 \mathrm{~m}$ & $29-\mid X-2007$ & Jose Manuel Sesma & Foto \\
\hline 31TDF28 & Barcelona & Esplugues de Llobregat & $95 \mathrm{~m}$ & $12-X-2007$ & Divina Aparicio & Foto \\
\hline 31TDG25 & Barcelona & Sant Martí d'Albars & $605 \mathrm{~m}$ & $17-\mathrm{VIII}-2009$ & Jordi Clavell & Foto \\
\hline 31TDF59 & Barcelona & Cabrils & $11 \mathrm{~m}$ & $11-\mathrm{Vl}-2011$ & Anna Gallés & Foto \\
\hline 31TDF16 & Barcelona & Gavà & $2 \mathrm{~m}$ & $9-X-2011$ & Ferran Turmo & Foto \\
\hline 31TCF96 & Barcelona & Villanueva y Geltrú & $30 \mathrm{~m}$ & $4-V-2012$ & Antoni Garcia Llorca & Foto $^{(2)}$ \\
\hline 31TDF18 & Barcelona & Molins de Rei & $24 \mathrm{~m}$ & 13-VII-2015 & Joaquim Morató & Foto \\
\hline 31TDG06 & Barcelona & Cercs & $718 \mathrm{~m}$ & 8-IX-2015 & Joan Escobet & Foto \\
\hline 31TDF27 & Barcelona & Sant Joan Despí & $21 \mathrm{~m}$ & $7-X I-2015$ & Martí Pérez & Foto \\
\hline 31TDF38 & Barcelona & Badalona & $32 \mathrm{~m}$ & $28-\mathrm{VII}-2016$ & Josefina Miralles & Foto \\
\hline 31TDG05 & Barcelona & Avià & $601 \mathrm{~m}$ & $5-I X-2016$ & Joan Escobet & Foto \\
\hline 31TDG00 & Barcelona & Collbató & $509 \mathrm{~m}$ & $2-I X-2018$ & Ferran Lizana & Foto \\
\hline 30SUJ08 & Cáceres & Navatrasierra & $722 \mathrm{~m}$ & $24-V I-2019$ & Manuel Moro & Foto \\
\hline 30TVP41 & Cantabria & Galizano & $13 \mathrm{~m}$ & $2-\mathrm{VII}-2010$ & Javier Soto & Foto \\
\hline 30TVP31 & Cantabria & Santander & $21 \mathrm{~m}$ & $16-V-2019$ & Oficina Ecocampus & Foto \\
\hline $30 S Y K 40$ & Castellón & Almenara & $3 \mathrm{~m}$ & $26-I V-2011$ & Rafael Pardo & Foto \\
\hline 30SYK11 & Castellón & Navajas & $389 \mathrm{~m}$ & $17-\mathrm{VIIII-2018}$ & Juan Ramón Vázquez & Foto \\
\hline 31TBE76 & Castellón & Peñíscola & $4 \mathrm{~m}$ & $10-\mathrm{XI}-2018$ & Francisco Arnau & Foto \\
\hline 31TEG14 & Gerona & Playa de Pals & $1 \mathrm{~m}$ & 2-VIIII-1995 & Salvador V. Peris & Captura $^{(3)}$ \\
\hline 31TDG72 & Gerona & Maçanet de la Selva & $70 \mathrm{~m}$ & $11-V-1998$ & André van Eck & Captura ${ }^{(4)}$ \\
\hline 31TEG16 & Gerona & L'Escala & $5 \mathrm{~m}$ & $27-V-1998$ & Tschorsnig & Captura ${ }^{(4)}$ \\
\hline
\end{tabular}


Table 1. - (Continued)

\begin{tabular}{|c|c|c|c|c|c|c|}
\hline \multicolumn{7}{|c|}{ ESPAÑA } \\
\hline UTM & Provincia & Localidad & Altitud & Fecha & Autoría & Fuente \\
\hline 31TDG77 & Gerona & Sales de Llierca & $271 \mathrm{~m}$ & $20-\mathrm{VI}-2007$ & Rafael Carbonell & Captura(5) \\
\hline 31TEG07 & Gerona & Sant Pere Pescador & $3 \mathrm{~m}$ & $23-X-2011$ & Vicent Romero & Foto \\
\hline 31TDG81 & Gerona & Lloret de Mar & $22 \mathrm{~m}$ & 9-VIII-2013 & Martin Reinhard Hoffmann & Foto \\
\hline $31 \mathrm{TDG} 46$ & Gerona & Vidrà & $1008 \mathrm{~m}$ & 23-VIII-2016 & Jordi Clavell & Foto \\
\hline 31TDG73 & Gerona & Santa Coloma de Farnés & $140 \mathrm{~m}$ & $10-I X-2017$ & Orlando Ferguson & Foto \\
\hline 31TDG86 & Gerona & Banyoles & $181 \mathrm{~m}$ & $18-\mid X-2018$ & Jordi Castellet & Foto \\
\hline 30SVF56 & Granada & Motril & $2 \mathrm{~m}$ & $4-\mathrm{VIII}-2013$ & Fernando Navarrete & Foto \\
\hline 30SVF58 & Granada & Talará & $710 \mathrm{~m}$ & 30-VIII-2016 & Guillermo Booth & Foto \\
\hline 30SVF89 & Granada & Yegen & $1056 \mathrm{~m}$ & 8-V-2019 & Simon Oliver & Foto \\
\hline 31TBG87 & Huesca & Graus & $472 \mathrm{~m}$ & $26-\mathrm{VII}-2013$ & Carlos González & Foto \\
\hline 31TBG75 & Huesca & Fonz & $503 \mathrm{~m}$ & $1-V-2014$ & Javier Cambra & Foto \\
\hline 31TBG56 & Huesca & Castillazuelo & $371 \mathrm{~m}$ & $2-\mathrm{VIII}-2018$ & Pedro Antonio Lázaro & Foto \\
\hline 30TYM18 & Huesca & Nueno & $1024 \mathrm{~m}$ & $20-\mathrm{VI}-2019$ & Cor Zonneveld & Foto $^{(6)}$ \\
\hline 31TBF59 & Huesca & Candasnos & $284 \mathrm{~m}$ & $19-\mathrm{VII}-2019$ & Sandy Rae & Foto $^{(7)}$ \\
\hline 29TNH69 & La Coruña & Betanzos & $34 \mathrm{~m}$ & $11-X-2011$ & Belén Amarante & Foto \\
\hline 31TCG00 & Lérida & Lérida & $208 \mathrm{~m}$ & $18-\mid X-2009$ & J. Ignasi Tejedor & Foto \\
\hline 31TCG34 & Lérida & Alòs de Balaguer & $318 \mathrm{~m}$ & $5-V-2018$ & Ferran Turmo & Foto \\
\hline 30TVK65 & Madrid & Perales de Tajuña & $622 \mathrm{~m}$ & 22-VIII-2018 & Enrique Hernández & Foto \\
\hline 30SXG79 & Murcia & Sierra de Columbares & $222 \mathrm{~m}$ & $11-\mid X-2010$ & Antonio Robledo & Foto \\
\hline 30SXG49 & Murcia & Librilla & $176 \mathrm{~m}$ & $29-V-2011$ & Emilio Alejandro Pagán & Foto \\
\hline 30SWH82 & Murcia & Moratalla & $1075 \mathrm{~m}$ & $19-\mathrm{VI}-2014$ & Antonio Robledo & Foto \\
\hline 29TNG26 & Pontevedra & Gondomar & $42 \mathrm{~m}$ & $13-\mathrm{VI}-2010$ & Javier Ferreiro & Foto \\
\hline 29TNG17 & Pontevedra & Donón (Cangas) & $16 \mathrm{~m}$ & $19-\mathrm{VII}-2015$ & Jesús Tizón & Foto \\
\hline 29TNG36 & Pontevedra & Ribadelouro (Tui) & $13 \mathrm{~m}$ & $3-X-2015$ & Jesús Tizón & Foto \\
\hline 30STG67 & Sevilla & Villanueva del Río y Minas & $40 \mathrm{~m}$ & $26-\mathrm{VIII}-2007$ & Diego Conradi & Foto \\
\hline 30STG53 & Sevilla & Alcalá de Guadaira & $48 \mathrm{~m}$ & 27-VIII-2008 & Diego Conradi & Foto \\
\hline 29SQB42 & Sevilla & Aznalcazar & $31 \mathrm{~m}$ & 17-IV-2009 & Diego Conradi & Foto \\
\hline 30STG36 & Sevilla & Burguillos & $48 \mathrm{~m}$ & $10-\mathrm{VIII}-2016$ & Fernando Laguna & Foto \\
\hline 30STG34 & Sevilla & Sevilla & $11 \mathrm{~m}$ & 23-IX-2014 & Fernando Laguna & Foto \\
\hline 29SQB45 & Sevilla & Gerena & $51 \mathrm{~m}$ & $27-\mid X-2011$ & Fernando Laguna & Foto \\
\hline 31TCF55 & Tarragona & Tarragona & $26 \mathrm{~m}$ & $24-X-2008$ & Ricard-Lluis Castell & Foto \\
\hline 31TCF56 & Tarragona & El Rourell & $116 \mathrm{~m}$ & $28-\mid X-2011$ & Josep María Solé & Foto \\
\hline 31TCF86 & Tarragona & Segur de Calafell & $40 \mathrm{~m}$ & $26-I X-2013$ & Paul Muller & Foto \\
\hline 31TBE89 & Tarragona & Alcanar & 100 & 30-III-2016 & Greg Grzegorz Sobieraj & Foto $^{(2)}$ \\
\hline 31TCF35 & Tarragona & Reus & $114 \mathrm{~m}$ & 13-VIII-2018 & Josep Roig & Foto \\
\hline 30SYJ29 & Valencia & Náquera & $240 \mathrm{~m}$ & $6-\mathrm{VI}-2005$ & Catalán y Verdú & Captura ${ }^{(8)}$ \\
\hline 30SYJ35 & Valencia & El Saler & $5 \mathrm{~m}$ & $5-V-2007$ & Rafael Pardo & Foto \\
\hline 30SYJ11 & Valencia & Canals & $155 \mathrm{~m}$ & $20-I X-2007$ & Fani Martínez & Foto \\
\hline $30 S \times J 98$ & Valencia & Pedralba & $206 \mathrm{~m}$ & 13-VIII-2009 & Fran Vives & Foto \\
\hline $30 S Y J 27$ & Valencia & Benimamet & $42 \mathrm{~m}$ & $25-I V-2011$ & Luis Vivas & Foto \\
\hline 30SYJ19 & Valencia & Olocau (Valencia) & $280 \mathrm{~m}$ & $14-I V-2013$ & Jorge Pérez & Foto \\
\hline $30 S Y J 26$ & Valencia & Tancat de la Pipa & $2 \mathrm{~m}$ & $1-\mathrm{VII}-2017$ & Pablo Vera & Foto \\
\hline 30SYJ17 & Valencia & Paterna & $61 \mathrm{~m}$ & $19-I V-2018$ & Juan Carlos Galey & Foto \\
\hline 30SYJ10 & Valencia & Carricola & $302 \mathrm{~m}$ & 7-VIIII-2018 & Fernando García & Foto \\
\hline 30SYJ39 & Valencia & Sagunto & $30 \mathrm{~m}$ & 29-III-2019 & juanvi & Foto $^{(7)}$ \\
\hline 30SYJ38 & Valencia & Valencia & $1 \mathrm{~m}$ & $21-\mid X-2019$ & Juan Ramón Vázquez & Foto \\
\hline 30TVN99 & Vizcaya & Getxo & $65 \mathrm{~m}$ & $23-\mid X-2011$ & Juancar Diéguez & Foto \\
\hline зотХМ71 & Zaragoza & Zaragoza & $225 \mathrm{~m}$ & $10-X-2008$ & José Luis Mateo & Foto \\
\hline зотХM92 & Zaragoza & Perdiguera & $469 \mathrm{~m}$ & $13-I X-2010$ & Constantino Escuer & Foto \\
\hline зотХМ82 & Zaragoza & Montañana & $231 \mathrm{~m}$ & $7-\mid X-2013$ & Henri Bourrut Lacouture & Foto \\
\hline зотХМ81 & Zaragoza & Movera & $189 \mathrm{~m}$ & 26-VIII-2015 & Henri Bourrut Lacouture & Foto \\
\hline
\end{tabular}


Table 1. - (Continued)

\begin{tabular}{|c|c|c|c|c|c|c|}
\hline \multicolumn{7}{|c|}{ PORTUGAL } \\
\hline UTM & Distrito & Localidad & Altitud & Fecha & Autoría & Fuente \\
\hline 29TNF31 & Aveiro & Estarreja & $10 \mathrm{~m}$ & $4-\mathrm{VIII}-2010$ & Rui Andrade & Captura $^{(9)}$ \\
\hline 29TNF29 & Braga & Barcelos & $40 \mathrm{~m}$ & $29-\mid X-2008$ & Rui Andrade & Foto $^{(9)}$ \\
\hline 29TPE12 & Castelo Branco & Almaceda & $340 \mathrm{~m}$ & $21-\mathrm{VIII}-2010$ & Jorge Mota Almeida & Foto $^{(9)}$ \\
\hline 29SPB22 & Faro & Azinhal & $80 \mathrm{~m}$ & $25-\mathrm{VII}-2011$ & Valter Jacinto & Foto $^{(9)}$ \\
\hline 29SNB18 & Setúbal & Porto Covo & $5 \mathrm{~m}$ & 8-VIII-2008 & Joaquim Gaspar & Foto $^{(10)}$ \\
\hline 29TNG11 & $\begin{array}{l}\text { Viana do } \\
\text { Castelo }\end{array}$ & Viana do Castelo & $6 \mathrm{~m}$ & $27-V-2017$ & Pedro Gomes & Foto \\
\hline
\end{tabular}

dentro de una misma cuadrícula UTM de $10 \times 10$, en la Tabla 1 se refleja el dato más antiguo, con el fin de tener información aproximada de la expansión de la especie. Además, en la Tabla 1 se recogen separadamente los datos de España y Portugal, con indicación, en cada caso, de la cuadrícula UTM, la provincia o distrito y la localidad donde se encontró el ejemplar, la altitud aproximada de la localidad (en m.s.n.m), la fecha de la observación y la autoría de cada cita, así como la fuente del registro. Las cuadrículas se ordenaron por fecha de observación dentro de cada provincia o distrito y estos, a su vez, alfabéticamente. La mayor parte de los datos proceden de la plataforma Biodiversidad Virtual. Con todos los datos de la Tabla 1, se preparó un mapa de distribución de T. pennipes en la Península Ibérica y Baleares (Fig. 3). El mapa fue elaborado con el programa QGIS 3.2.0.

Con el fin de poder comparar la distribución ibero-balear de T. pennipes con la de su principal huésped, $N$. viridula, se aportó la representación de los datos georreferenciados del Banco Taxonómico de Biodiversidad Virtual para esta especie, en términos de densidad, con el número de registros por cuadrícula UTM de $10 \times 10 \mathrm{~km}$ (mapa descargado de la

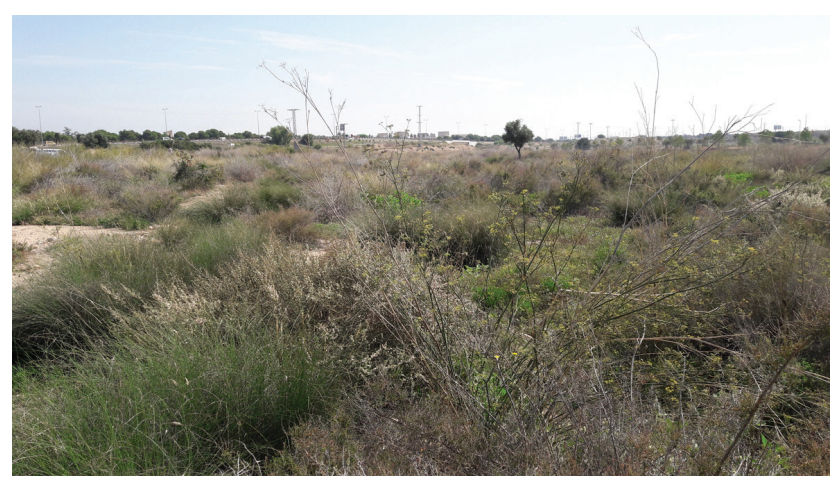

Fig. 2.- Localidad donde se recolectó Trichopoda pennipes (Fabricius, 1781) en el municipio de Alicante, en octubre de 2019 (foto: A. Ricarte).

Fig. 2.- Collecting site of Trichopoda pennipes (Fabricius, 1781 ) in the municipality of Alicante in October 2019 (photo: A. Ricarte). plataforma como archivo kml) (Fig. 4). La comparación se hizo cualitativamente, mediante observación de la presencia/ausencia o cantidad relativa de registros confirmados de una y otra especie en una misma parte del mapa. Adicionalmente, se aportó el mapa de densidad de las citas de insectos recogidos en el Banco Taxonómico de dicha plataforma (obtenido de manera análoga al anterior), y que refleja la intensidad de muestreo fotográfico de los usuarios de Biodiversidad Virtual (Fig. 5).

Finalmente, y dado que se disponía de 134 citas, tanto bibliográficas como fotográficas, con datos completos dentro del territorio ibero-balear, se realizó un análisis de la fenología de T. pennipes dentro de este. Para ello, se contabilizó el número de citas de T. pennipes asociado a cada mes del año y dichos números se representaron en un histograma con los 12 meses (Fig. 6), mediante el programa Excel de Microsoft Office ${ }^{\circledR}$.

\section{Resultados}

Se recolectaron un macho y una hembra de T. pennipes junto al recinto del Consorcio Provincial para el Servicio de Prevención y Extinción de Incendios y Salvamento de Alicante $\left(38^{\circ} 23^{\prime} 0.15^{\prime \prime} \mathrm{N}\right.$ $0^{\circ} 31^{\prime 26.3 " O}, 87 \mathrm{~m}$ ), en el municipio homónimo, el 1 de octubre de 2019. Los ejemplares fueron encontrados en un campo nitrófilo con vegetación ruderal (p. ej., Atriplex y Salsola) y propia de bordes de caminos (p. ej., Foeniculum vulgare y Ecballium) (Fig. 2). La constatación de la ausencia previa de esta especie en la provincia de Alicante, así como la escasez de citas ibero-baleares, de acuerdo a la bibliografía consultada, motivó la búsqueda de registros adicionales en bases de datos en línea. En dicha búsqueda, apareció un notable número de cuadrículas UTM en las que no estaba documentada previamente la presencia de esta especie (incluidas otras citas en la provincia de Alicante), incrementándose así el nivel de conocimiento de su distribución en el ámbito ibero-balear (Tabla 1). En el mapa obtenido con los datos de la Tabla 1, queda patente que los nuevos registros de la 


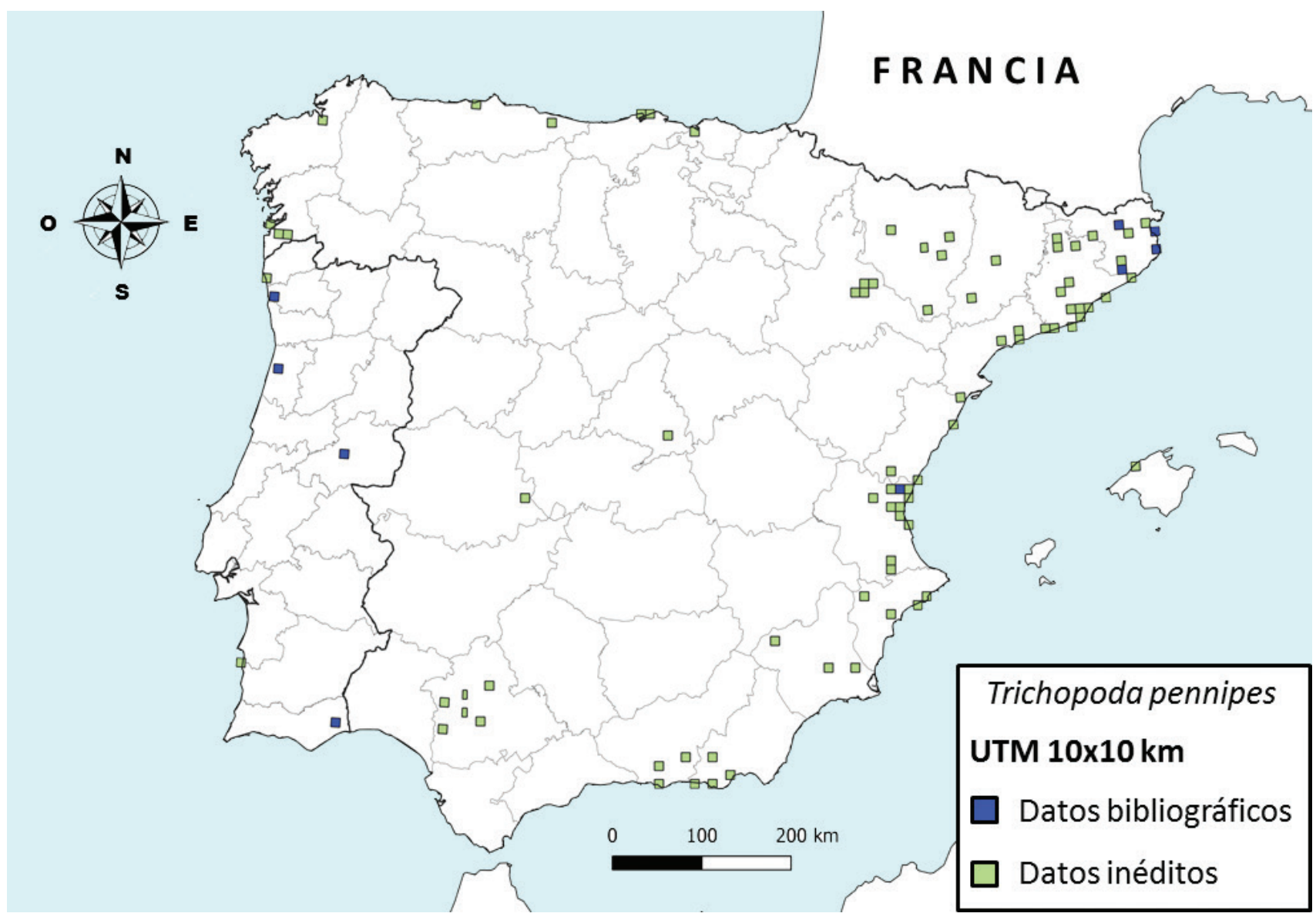

Fig. 3.- Mapa de distribución de Trichopoda pennipes (Fabricius, 1781) en la Península lbérica e Islas Baleares, con todas las cuadrículas UTM $10 \times 10 \mathrm{~km}$ confirmadas hasta la fecha.

Fig. 3.- Range map of Trichopoda pennipes (Fabricius, 1781) in the Iberian Peninsula and Balearic Islands, including all confirmed $10 \times 10-\mathrm{km}$ UTM squares to date.

especie son más numerosos que los registros bibliográficos, existiendo una sola cita confirmada en Baleares (Fig. 3). Además, la mayoría de los registros se hallan cerca, o relativamente cerca, de la costa, siendo especialmente numerosos en el Mediterráneo. En la zona central de la Península, aparecen escasamente dos citas. También se observan zonas de concentración de datos, por ejemplo, en torno a la capital valenciana (Fig. 3). Por otro lado, la gráfica de fenología muestra que los picos de actividad del adulto de $T$. pennipes se presentan en agosto y septiembre, mientras que no existen registros en diciembre, enero y febrero (Fig. 6).

\section{Discusión}

El estudio de T. pennipes en la región ibero-balear entraña un interés particular, puesto que se trata de una especie exótica introducida y, al mismo tiempo, parasitoide de hemípteros plaga como $N$. viridula. La revisión de fuentes bibliográficas y bases de datos de internet, realizada tras el hallazgo de adultos de T. pennipes en un erial de Alicante, ha arrojado abundantes resultados novedosos, muchos de ellos trasladados desde el ámbito de la llamada ciencia ciudadana al ámbito académico, mediante el presente trabajo. Estos datos, de índole principalmente geográfica y fenológica, han incrementado considerablemente el conocimiento ibero-balear de la especie con respecto al plasmado, hasta la fecha, en artículos científicos. La mayoría de datos manejados en este estudio proceden de evidencias fotográficas y convierten a la Península Ibérica en la región de Europa donde mejor se conoce la distribución geográfica y temporal de T. pennipes.

El presente trabajo aporta una notable cantidad de cuadrículas UTM donde se confirma la presencia de $T$. pennipes, siendo inéditos los datos de 80 de las 89 cuadrículas estudiadas (Fig. 3). El 90\% de las cuadrículas inéditas proceden de datos de BiodiversidadVirtual.org. Los únicos datos bibliográficos existentes sobre T. pennipes en España eran de ejemplares recolectados en los años 1995 y 1998, en la provincia de Gerona (Peris, 1998; Tschorsnig et al., 2000), y en el año 2005, en la provincia de Valencia (Catalán \& Verdú, 2005), pero las citas fotográficas actuales parecen indicar que la especie ha colonizado buena parte de la Península; interesa destacar que no es esta la primera vez que un artículo científico aporta 
registros de $T$. pennipes basados en material fotográfico (Carbonell, 2013; Almeida et al., 2017). La cita fotográfica más antigua es del año 2007 y procede de Sevilla. Por su parte, las dos citas (fotográficas) aisladas del interior peninsular (Fig. 3) son muy recientes, de 2018 (Madrid) y 2019 (Cáceres).

En España, la presencia de T. pennipes se concentra fundamentalmente en la vertiente mediterránea, existiendo citas más dispersas en la zona costera cantábrica y en el interior peninsular (Fig. 3, Tabla 1). Por su parte, los registros portugueses (Almeida et al., 2017, presente trabajo) no parecen concentrarse en una parte específica del país, pues hay datos de zonas cercanas a la costa, otros de zonas interiores y también tanto al norte como al sur del país (Fig. 3, Tabla 1). Aparentemente, existe una mayor abundancia de T. pennipes en zonas costeras de España que en interiores, no habiendo registros en áreas montañosas elevadas (más de $1100 \mathrm{~m}$ de altitud) y siendo estos escasos a altitudes intermedias (500-1100 m), a las cuales solo se han detectado 11 poblaciones (Fig. 3, Tabla 1).
El bajo número de registros de $T$. pennipes en el interior peninsular parece reflejar una verdadera escasez de la especie en esta zona, pues los mapas de densidad de citas totales de usuarios de BiodiversidadVirtual. org (Fig. 5) indican una intensa actividad fotográfica en zonas interiores, que, sin embargo, no ha revertido proporcionalmente en el número de registros de T. pennipes; véase el caso de la provincia de Madrid, en la que solo hay un registro de esta especie (Fig. 3). No obstante, sería necesario un estudio comparativo de la presencia de la especie en diferentes zonas, basado en campañas de muestreo/monitoreo y análisis estadísticos de los resultados para corroborar estas observaciones.

La mayor densidad de citas de T. pennipes en zonas costeras y la aparente agregación de las mismas, con zonas de menor densidad entre agregados, podría estar relacionada con la entrada de la especie vía portuaria, en mercancías hortofrutícolas frescas portadoras de $N$. viridula parasitoidizada por $T$. pennipes. Esto explicaría, por ejemplo, los agregados de citas

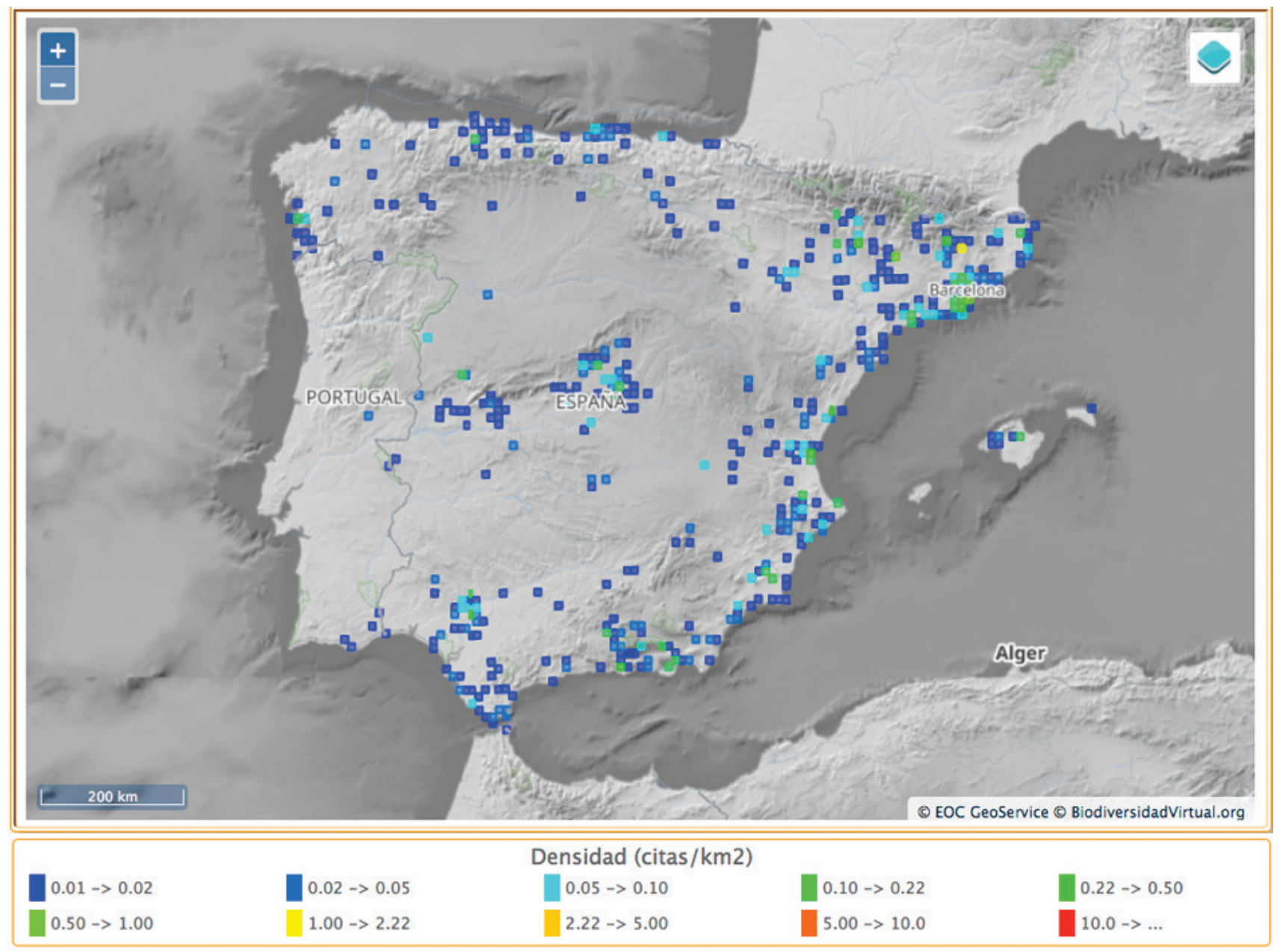

Fig. 4.- Mapa de distribución de los registros fotográficos de Nezara viridula (Linnaeus, 1758) disponibles en la plataforma Biodiversidad Virtual.

Fig. 4.- Distribution map of the photographic records of Nezara viridula (Linnaeus, 1758) available at the website 'Biodiversidad Virtual'. 


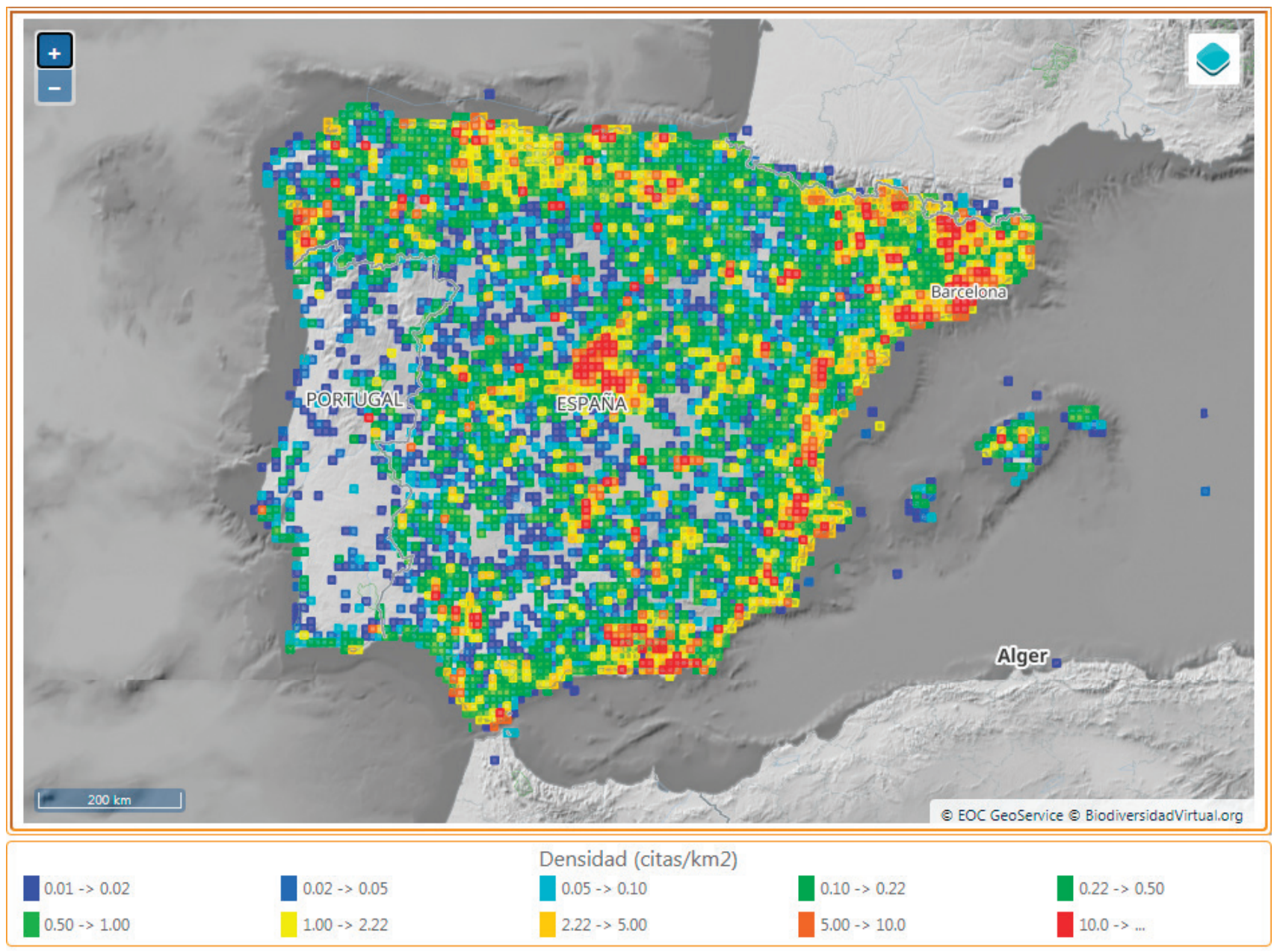

Fig. 5.- Mapa de las citas fotográficas de insectos recogidas en el Banco Taxonómico de la plataforma Biodiversidad Virtual, mostrado la intensidad de muestro de los usuarios de dicha plataforma.

Fig. 5.- Map of the photographic records of insects gathered in the Taxonomic Bank of the website 'Biodiversidad Virtual', showing the intensity of sampling of this website's users.

cercanos a los puertos de Barcelona y Valencia (Fig. 3). La entrada de T. pennipes a un país europeo a través de intercambio de mercancías ya ha sido reportada por otros autores, pero se refiere a intercambio de mercancías en aeropuertos (Colazza et al., 1996). Valencia y Barcelona, sobre todo la segunda, también tienen importantes aeropuertos que podrían haber sido vía de entrada de T. pennipes, pero Madrid carece, por el momento, de registros de la especie cercanos a su aeropuerto, pues Perales de Tajuña, donde se ha fotografiado T. pennipes (Tabla 1), se encuentra a más de $30 \mathrm{~km}$ del aeropuerto de Madrid. La elevada actividad hortofrutícola en ciertas zonas también es motivo potencial de una mayor presencia de $T$. pennipes, pues estas zonas tienen, a priori, más abundancia de Nezara, que es su huésped (Peris, 1998); sería el caso de la ciudad de Valencia y alrededores (Fig. $3)$. Del mismo modo, el menor número de registros de $T$. pennipes en la cornisa cantábrica podría correlacionarse con una menor presencia de cultivos hortofrutícolas en esta zona y, por tanto, una menor abundancia de sus huéspedes. Esta posible - aunque también lógica - correlación entre la presencia de T. pennipes y sus huéspedes parece verse avalada al compararse los mapas de distribución de $T$. pennipes (Fig. 3) y N. viridula (Fig. 4), en los que, por ejemplo, se aprecian altas concentraciones de $N$. viridula en torno a la ciudad de Barcelona, donde también se da un agregado de citas de T. pennipes. También se observan amplias zonas de ausencia o escasez simultáneas de ambas especies, por ejemplo, en Castilla-yLeón y Castilla-La-Mancha. Trichopoda pennipes ha sido capaz de colonizar con éxito regiones tan dispares, ambientalmente hablando, como Hawaii (Hardy, 1981) o Eslovenia (de Groot et al., 2007) y, por tanto, descartamos un efecto marcado de las condiciones climáticas en la distribución ibero-balear de esta especie, siendo la disponibilidad de huéspedes, muy probablemente, el factor limitante en este sentido.

Como se puede observar en la fenología de T. pennipes (Fig. 6), en el área de estudio, esta es una especie que vuela durante la primavera y el verano, con el pico en los meses de agosto y septiembre, si bien su época de vuelo puede alargarse, con citas muy 


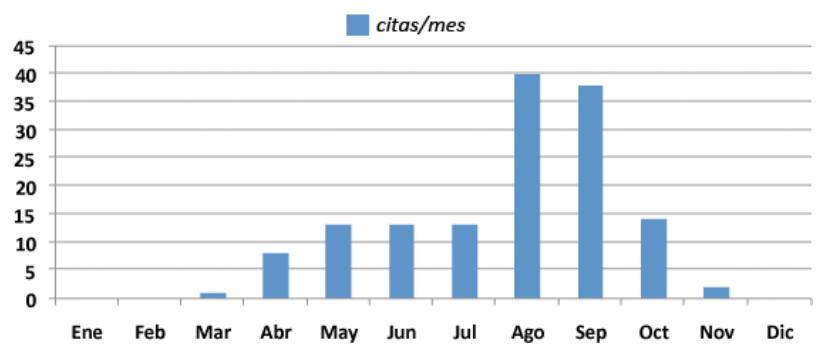

Fig. 6.- Fenología de Trichopoda pennipes (Fabricius, 1781) en la Península lbérica y Baleares, basada en las 134 citas recopiladas tanto de ejemplares capturados como de fotografías.

Fig. 6.- Phenology of Trichopoda pennipes (Fabricius, 1781) in the Iberian Peninsula and Balearic Islands, based on the 134 records gathered from captured specimens and photographs.

tempranas, a finales de marzo y tardías, a principios de noviembre. Estos datos parecen indicar que, al igual que en el Nuevo Mundo (Pétremand et al., 2015), presenta, al menos, dos generaciones al año. Otro aspecto que interesa mencionar es el tipo de plantas que visita fuera de su área de origen. Trichopoda pennnipes podría comportarse como un potencial polinizador generalista, según se concluye de las imágenes estudiadas de los imagos libando en diferentes plantas, a partir de las cuales también se concluye que este taquínido se alimenta de néctar de plantas ibéricas pertenecientes a familias presentes en el Nuevo Mundo, como Apiaceae (Crithmum maritimum, Eryngium campestre y Foeniculum vulgare), Asteraceae (Achillea millefolium, Helichrysum stoechas, Inula viscosa), Boraginaceae (Anchusa sp., Heliotropium europaeum) y Lamiaceae (Mentha sp.), entre otras.

Por último, algunos autores alertan del peligro que la introducción de $T$. pennipes puede tener para la fauna nativa de hemípteros (de Groot et al., 2007; Fand et al., 2013), en función de su capacidad para atacar tanto a ninfas como adultos de chinches (Catalán \& Verdú, 2005) y de los ataques registrados a especies nativas de pentatómidos en ciertas partes del mundo donde ha sido introducida, por ejemplo, en Hawaii (Johnson et al., 2005). No obstante, la baja fecundidad de los Phasiinae (Marshall, 2012) unida al hecho que, hoy por hoy, no existen evidencias publicadas de daños a especies nativas por parte de T. pennipes en Europa, sugieren que su impacto en la biodiversidad nativa puede ser bajo o, como mucho, moderado. Para conocer el efecto real de esta especie en los ecosistemas ibéricos, en primer lugar, es necesario confirmar si los registros aislados representan focos independientes de introducción o, simplemente, la especie está extendida por toda la Península y sigue habiendo amplios vacíos de conocimiento, lo cual parece improbable a la vista de los resultados aquí discutidos. En este sentido, este estudio puede servir de marco básico para tratar de detectar este taquínido en las amplias zonas peninsulares donde todavía no se ha registrado. En segundo lugar, es indispensable realizar estudios de campo para averiguar cuáles son los hemípteros huésped de $T$. pennipes en esta región geográfica y qué impacto tiene en ellos. Afortunadamente, ninguna de las familias a las que pertenecen los huéspedes de T. pennipes Pentatomidae, Coreidae, Pyrrhocoridae y Alydidae (Catalán \& Verdú, 2005) — está recogida en el Libro Rojo de los Invertebrados de España (Verdú \& Galante, 2005).

A la vista de los resultados publicados en este trabajo, nuevamente, se pone de manifiesto la importancia que los bancos de datos fotográficos tienen en el conocimiento de la biodiversidad, sobre todo en el caso de las especies más singulares (Marcos-García \& Ricarte, 2012) y fácilmente distinguibles de otras (Álvarez Fidalgo et al., 2018).

\section{Agradecimientos}

Agradecemos al Dr. Eduardo Galante Patiño su opinión taxonómica, a nivel familia, sobre los ejemplares recolectados y a Gabriel Souba Dols su ayuda en la caracterización del hábitat donde se recolectó T. pennipes en Alicante. A Ángel Mario Moreno agradecemos la cesión de su fotografía de una hembra de Trichopoda pennipes para este artículo y a la plataforma Biodiversidad Virtual su colaboración para que este trabajo sea una realidad, así como a los otros especialistas de la plataforma que han contribuido a la identificación de las fotografías (Miguel Carles Tolrá, Paqui Mars y Ana Cobo) la valiosa labor realizada desinteresadamente. También queremos expresar nuestro agradecimiento a todos los naturalistas que, altruistamente, comparten sus datos y fotografías de campo en las plataformas ciudadanas, por su contribución al conocimiento científico. El contrato de Antonio Ricarte (Ref. UTAL05) en la Universidad de Alicante está financiado por el Vicerrectorado de Investigación y Transferencia de Conocimiento.

\section{Referencias}

Almeida, J. M., Andrade, R., Gonçalves, A., Jacinto, V., Raper, C., Tschorsnig, H.-P. \& Zeegers, T., 2017. An annotated checklist of the Tachinidae (Diptera) of mainland Portugal. Boletín de la Sociedad Entomológica Aragonesa, 60: 62-76.

Álvarez Fidalgo, M., Álvarez Fidalgo, P., Ricarte Sabater, A. \& Marcos García, M. A., 2018. The genus Leucozona Schiner, 1860 on the Iberian Peninsula, including the first records of Leucozona laternaria (Müller, 1776) (Diptera: Syrphidae). BV News Publicaciones Cientificas, 7(98): 128-141.

Biodiversidad Virtual, 2020. Base de datos en línea. Disponible en: https://www.biodiversidadvirtual.org. Acceso: 18 Feb. 2020.

Bystrowski, C., 2012. Thoughts on how Trichopoda pennipes (F.) reached Israel. The Tachinid Times, 25: 13-15.

Carbonell, R., 2013. Artròpodes aliens de la Garrotxa: Harmonia axyridis (Insecta: Coleoptera), nova espècie invasora i actualització de la llista d'artròpodes forans 
de la Garrotxa. Annals de la delegació de la Garrotxa de la Institució Catalana de Història Natural, 6: 65-75.

Catalán, J. \& Verdú, M. J., 2005. Dispersión de Trichopoda pennipes (Fabricius, 1781) (Diptera: Tachinidae) parasitoide de Nezara viridula (L.) en el área mediterránea. Boletín de la Asociación española de Entomología, 29(3-4): 127-128.

Colazza, S., Giangiuliani, G. \& Bin, F., 1996. Fortuitous introduction and successful establishment of Trichopoda pennipes F.: adult parasitoid of Nezara viridula (L.). Biological Control, 6: 409-411. https://doi. org/10.1006/bcon.1996.0053

de Groot, M., Virant-Doberlet, M. \& Žunič, A., 2007. Trichopoda pennipes F. (Diptera, Tachinidae): A new natural enemy of Nezara viridula (L.) in Slovenia short communication. Agricultura, 5(1): 25-26.

Diptera.info, 2020. [en línea] Disponible en: https://diptera. info/news.php. Acceso: 18 Feb. 2020.

Fand, B. B., Suroshe, S. S. \& Gautam, R. D., 2013. Fortuitous biological control of insect pests and weeds: a critical review. The Bioscan, 8(1): 1-10.

Freidberg, A., Morgulis, E. \& Cerretti, P., 2011. The invasive species, Trichopoda pennipes (F.) (Diptera: Tachinidae), found in Israel. The Tachinid Times, 24: 14-15.

GBIF, 2020. Global Biodiversity Information Facility in Spain. Nodo Nacional de Información en Biodiversidad. [en línea] Disponible en: http://datos.gbif.es/. Acceso: 20 Feb. 2020.

Grenier, S., 1988. Applied biological control with Tachinid flies (Diptera, Tachinidae): a review. Anzeiger für Schädlingskunde, Pflanzenschutz, Umweltschutz, 61: 49-56.

iNaturalist, 2020. Base de datos en línea. [en línea] Disponible en: https://www.inaturalist.org. Acceso: 18 Feb. 2020.

Hardy, E., 1981. Insects of Hawaii, Vol. 14. Diptera: Cyclorrapha IV, Series Schizophora, Section Calyptratae. The University Press of Hawaii. Honolulu. 491 pp.

Johnson, M. T., Follett, P. A., Taylor, A. D. \& Jones, V. P., 2005. Impacts of biological control and invasive species on a non-target native Hawaiian insect. Oecologia, 142(4): 529-540. https://doi.org/10.1007/ s00442-004-1754-5

Marcos-García, M. A. \& Ricarte, A., 2012. Segunda localidad ibérica conocida para Caliprobola speciosa (Rossi), especie UICN - "En peligro" (Diptera, Syrphidae). Boletín de la Asociación española de Entomología, 36(1-2): 207-210.

Marshall, S. A., 2012. Flies: the natural history and diversity of Diptera. Firefly Books Ltd. USA and Canada. $616 \mathrm{pp}$.

Observation.org, 2020. Base de datos en línea de acceso libre. [en línea] Disponible en: https://observation.org/. Acceso: 18 Feb. 2020.

O’Hara, J. E., 2008. Tachinid flies (Diptera: Tachinidae). In: Capinera, J.L. (ed.). Encyclopedia of Entomology, 2 ${ }^{\text {nd }}$ Edition: 3675-3686. Springer Netherlands. Dordrecht.

Peris, S. V., 1998. Un Trichopodini (Diptera, Tachinidae, Phasiinae) en España. Boletín de la Real Sociedad
Española de Historia Natural (Sección Biológica), 94(1-2): 163-164.

Pétremand, G., Rochefort, S., Jaccard, G. \& Fischer, S., 2015. First detection of the southern green stink bug parasitoid Trichopoda pennipes (Fabr.) (Diptera: Tachinidae) in Western Switzerland. Mitteilungen der Schweizerischen Entomologischen Gesellschaft, 88 (3-4): 403-409.

Pilkay, G. L., Reay-Jones, F. P. F. \& Greene, J. K., 2014. Host preference of the parasitoid Trichopoda pennipes (Diptera: Tachinidae) with Euschistus servus and Nezara viridula (Hemiptera: Pentatomidae). Journal of Entomological Science, 49(1): 56-62. https://doi. org/10.18474/0749-8004-49.1.56

Salerno, G., Colazza, S. \& Bin, F., 2002. Nezara viridula parasitism by the tachinid fly Trichopoda pennipes ten years after its accidental introduction into Italy from the New World. BioControl, 47(6): 617-624. https://doi. org/10.1023/A:1020521607677

Skuhravá, M., Martinez, M. \& Roques, A., 2010. Diptera. Chapter 10. In: Roques, A., Kenis, M., Lees, D., LopezVaamonde, C., Rabitsch, W., Rasplus, J.-Y. \& Roy, D. B. (Eds.). Alien terrestrial arthropods of Europe. BioRisk (Special Issue), 4: 553-602. https://doi.org/10.3897/ biorisk. 4.53

Stireman, III, J. O., O’Hara, J. E. \& Wood, D. M., 2006. Tachinidae: evolution, behavior, and ecology. Annual Review of Entomology, 51: 525-555. https://doi. org/10.1146/annurev.ento.51.110104.151133

Tillman, G., 2017. Ecosystem-based incorporation of nectar-producing plants for stink bug parasitoids. Insects, 8: 65-78. https://doi.org/10.3390/insects 8030065

Tooker, J. F., Hauser, M. \& Hanks, L. M., 2006. Floral host plants of Syrphidae and Tachinidae (Diptera) of Central Illinois. Annals of the Entomological Society of America, 99(1): 96-112. https:// doi.org/10.1603/0013-8746(2006)099[0096:FHPOS A]2.0.CO;2

Tschorsnig, H.-P. \& Richter, V.A., 1998. Family Tachinidae. In: Papp, L. \& Darvas, B. (Eds.). Contributions to a Manual of Palearctic Diptera (with special reference to flies of economic importance). Vol. 3: Higher Brachycera: 691-827. Science Herald. Budapest.

Tschorsnig, H.-P., Zeegers, T. \& Holstein, J., 2000. Nuevas citas del Noroeste de España y Sur de Francia del parasitoide Trichopoda pennipes (Fabricius, 1781) (Diptera, Tachinidae). Boletín de la Real Sociedad española de Historia Natural (Sección Biológica), 96(1-2): 215-216.

Verdú, J. R. \& Galante, E. (Eds.). 2005. Libro Rojo de los Invertebrados de España. Dirección General de Conservación de la Naturaleza. Madrid. Versión online: https:// cibio.ua.es/lrie/lrie.html

Worthley, H. N., 1924. The biology of Trichopoda pennipes Fab. (Diptera, Tachinidae), a parasite of the common squash bug. Part II. Morphology. Psyche: A Journal of Entomology, 31(2): 57-67. https://doi. org/10.1155/1924/96797

Zeegers, T., 2010. Tweede aanvulling op de naamlijst van Nederlandse sluipvliegen (Diptera: Tachinidae). Nederlandse Faunistische Mededelingen, 34: 55-66. 